\title{
A HYDROMAGNETIC MODEL FOR THE HIERARCHICAL STRUCTURE OF MOLECULAR CLOUDS
}

\author{
B.M. DEISS \\ Inst. f. Theor. Physik, Goethe-Universität Frankfurt, \\ 60054 Frankfurt, Germany \\ AND \\ A. JUST \\ Astronomisches Rechen-Institut, \\ 69120 Heidelberg, Germany
}

\section{Physical System and discrete 2-scale theory}

We propose a physical model of molecular clouds which is based on the idea that the back reaction of substructures of a cloud on the ambient medium maintains and stabilizes the cloud on larger scales: clumps, which are assumed to carry a magnetic moment, are coupled to the ambient medium by magnetic forces, hence continually inducing velocity fluctuations due to their random motion. The energy source is then the gravitational binding energy of the clumps in the global potential of the whole cloud.

On a given range of spatial scale we regard mol. clouds to be composed of: i) an ensemble of dense cores, the dynamics of which is described stellardynamically, and with the internal clump structure (mass, magnetic moment) held fixed; ii) a low density, partially ionized, twocomponent gas, the dynamics of which is treated by two-fluid magnetohydrodynamics, where we take ambipolar diffusion into account; iii) a magnetic field frozen in the ionized component. In quasi-linear approximation the power spectrum of the stationary turbulent MHD velocity field reads $C^{(2)}(k) \propto B_{c}^{2} n \sigma^{-1} D^{6} \chi k$, where $B_{c}, n, \sigma, D, \chi$ denote the clumps' magnetic field, number density, velocity dispersion and size, and the ionization degree of the ambient medium, respectively. $C^{(2)}$ increases with increasing $k$. Integrating $C^{(2)}(k)$ over $2 \pi / L \leq k \leq 2 \pi / D$, where $L$ denotes the size of the whole cloud, we obtain for the 1-dim. velocity dispersion 
$\left\langle V_{r}^{2}\right\rangle=\left(0.22 \frac{\mathrm{km}}{\mathrm{s}}\right)^{2}\left(\frac{B_{c}}{\mu \mathrm{G}}\right)^{4}\left(\frac{n}{\mathrm{pc}^{-3}}\right)\left(\frac{\sigma}{\mathrm{km} \mathrm{s}^{-1}}\right)^{-1}\left(\frac{\chi}{10^{-5}}\right)$. Examples: $\rho$-Oph $(1.1$ $\mathrm{km} / \mathrm{s})$; Taurus cloud $(0.42 \mathrm{~km} / \mathrm{s})$. This is in reasonable agreement with the observed values of $1.6 \mathrm{~km} / \mathrm{s}$ and $1.5 \mathrm{~km} / \mathrm{s}$, respectively.

\section{Hierarchical model and scaling relation}

Since clumps of a given size induce fluctuations on all larger scales, we regard $C^{(2)}(k)$ as a differential power spectrum excited by clumps $\mathrm{d} n$ of size $D$; i.e. $C^{(2)}(k) \rightarrow \mathrm{d} C(k, D)$. Integrating $\mathrm{dC}(\mathrm{k}, \mathrm{D})$ over all scales smaller than $k^{-1}$ yields the total spectrum $C(k) \propto k^{-e}$ with $e=6-c+s-2 d-a$, where we employed the observed scaling relations $\sigma \propto L^{a}, \mathrm{~d} n / \mathrm{d} L \propto L^{-c}$, $B \propto L^{-d}, \chi \propto L^{s}$. The turbulent velocity amplitude $\sigma_{\text {turb }}(L)$ on a given scale $L$ is given by the integral of $C(k)$ over all smaller scales $\pi / k \leq L$. Identifying the induced velocity fluctuations with the velocity dispersion of the substructures we find a selfconsistent power-law index $\alpha$ for the velocity dispersion $\sigma_{\text {turb }} \propto L^{\alpha}$ with $\alpha=(5-c+s-2 d) / 3$. From observations one infers $c \approx 2.3-3.4 ; s \approx 0.5-0.7 ; d \approx 0.3-0.7$. This yields $\alpha \approx$ $0.3-0.85$ which is in very good agreement with the observed scaling index $a \approx 0.3-0.7$.

\section{Stability of molecular clouds}

We put $p=p_{\text {therm }}+p_{\text {turb }}$ in Euler's equation and assume $p_{\text {turb }} \propto L^{2 a-b}$. The (linear) criterion for gravitational stability then reads $\left(4 \pi G \rho / c_{s}^{2}\right) \leq$ $k^{2}\left[1+\left(k / k_{e q}\right)^{1-e}(b-2 a) / a\right]$ with the normalization $\sigma_{t u r b}\left(k=k_{e q}\right)=c_{s}$. Since $e \approx 1.6-2.7$, mol. clouds are stable against large-scale collapse. However, there exists a finite range of scales $(\approx 1 \mathrm{pc})$ which still may be gravitationally unstable, hence leading to the formation of clumps and substructures.

\section{Conclusions}

Our magnetized-clump model demonstrates that it is just the clumpiness of mol. clouds which leads to turbulent motions and the large-scale stabilization of the clouds. The inferred scaling relation of the induced turbulent motions is determined by scaling relations of other physical parameters of mol. clouds. This is a first step to construct a physically coherent hierarchical model. The energy source in our model is the potential energy of the whole cloud; i.e., it is an internal source. Hence, the general, hierachical structure of mol. clouds and the turbulent motions within them, seem to be an intrinsic property of selfgravitating gaseous systems.

Ref.: A. Just, S. Jacobi, B.M. Deiss 1994, Astron.Astrophys. 289, 237 